\title{
ESTUDO RETROSPECTIVO TERAPÊUTICO DA NEUROCRIPTOCOCOSE EM 112 AIDÉTICOS OU NÃO
}

\author{
Evanil Pires de Campos, Valquíria Oliveira Carvalho, Sonia Fontes Marinho, \\ Tuba Milstein Kushnaroff, Paulo Augusto Ayrosa Galvão e Carlos Roberto \\ Padovani
}

\begin{abstract}
Cento e doze aidéticos ou não com neurocriptococose, admitidos no Hospital Emílio Ribas - São Paulo, Brasil, receberam anfotericina B (AMB) - grupo III ou a associação AMB/ 5 fluorcitosina (SFC): grupos I e II. Testes de Goodman aplicados revelaram: 1. leuco e glicorraquia semelhantes nos três grupos e proteinorraquia inferior a $85 \mathrm{mg} /$ dl lapós $1,5 \mathrm{~g} / \mathrm{AMB}$; 2. a coloração pelo método da tinta da China e a cultura para Cryptococcus neoformans, positivas até 1,Og/AMB; 3. hipocalemia na monoterapia, hipo e hipercalemia durante a

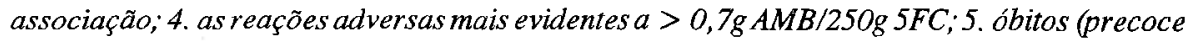
e tardio) frequentes no grupo I e nos grupos I III entre 2,5 a 4,Og de AMB; 6. remissão e morte semelhantes nos grupos. A associação terapêutica inicial e amanutenção pelo $A M B$ conduziram à recaída tardia
\end{abstract}

Palavras-chaves: Neurocriptococose. Aidéticos e não. Terapia.

I criptococose é doença causada pelo Cryptococcus neoformans que ao atingir os pulmôes, pela via inalatória, poderá disseminar-se ou não para diversos órgãos, tais como: S.R.E., adrenal, ossos e principalmente para o sistema nervoso central (SNC) 246789141521 .

A neurocriptococose é consequência habitual dessa doença fúngica e, para explicá-la, algumas especulações teóricas foram aventadas. A ocorrência da neurocriptococose em alcoólatras, diabéticos, no uso de corticóides e de neoplasias, realçou a importância desses fatores como predisponentes dessa micose profunda 3456791013 171820 .

A depleção de tiamina no alcoólatra, assim como a deficiência do triptofano poderiam facilitar o desenvolvimento da forma nervosa na criptococose 7121617182022 . Por outro lado, a ocorrência de neurocriptococose é frequente no doente aidético. Tal fato permitiu considerar a criptococose no SNC como doença oportunista nos aidéticos 26791320 .

Trabalho do Hospital Emilio Ribas, São Paulo e Instituto de Biociências, Universidade Estadual Paulista, Botucatu, SP. Enderego para correspondência: Prof. Evanil Pires de Campos. Rua João Miguel Rafael 350, 18602-220 Botucatu, SP, Brasil

Recebido para publicação em 16/03/92.
A terapia da criptococose apresenta controvérsia em relação à associação de antifúngicos e a monoterapia pela anfotericina $B(A M B)^{679132021}$.

A monoterapia pela $A M B$ é usualmente utilizada por algumas semanas e, a seguir, a terapia supressiva ou de manutenção é aplicada com o objetivo de se evitar a recaída da neurocriptococose.

A superioridade da associação AMB e 5 fluorcitosina (5FC) sobre a monoterapia (AMB) na esterilização liquórica, na menor nefrotoxicidade e na remissão clínica durante um período de 8 semanas foi verificado por Dismukes e coll $^{9}$. No entanto, em estudo similar Chuck e $\operatorname{col}^{6}$ não observaram diferença siginificativa na sobrevida de pacientes tratados pela associação de drogas (AMB e 5FC) e nos pacientes que utilizaram monoterapia (AMB).

Logo, a associação não propicia melhor resultado quando comparada à monoterapia pela AMB na neurocriptococose.

Pelo exposto, resolveu-se comparar os achados clínico-laboratoriais obtidos durante o uso da monoterapia com a associação antifúngica (AMB e $5 F C$ ), em estudo retrospectivo realizado em 112 pacientes com neurocriptococose, aidéticos ou não, internados no Hospital Emílio Ribas - São Paulo, com a finalidade de se verificar qual a conduta terapêutica mais propícia nesses doentes. 
Campos EP, Carvalho VO, Marinho SF, Kushnaroff TM, Galvão PAA, Pajovani CR. Estudo retrospectivo terapêutico da neurocriptococose em 112 aidéticos ou não. Revista da Sociedade Brasileira de Medicina Tropical 25:241-246, out-dez, 1992.

\section{MATERIAL E MÉTODOS}

Analisaram-se 112 pacientes, 86 aidéticos e 26 não aidéticos, com neurocriptococose confirmada à internação no Hospital Emílio Ribas - São Paulo, tratados pela AMB e pela associação AMB e SFC. a saber:

Os pacientes foram subdivididos em três grupos,

\section{Grupo I: 26 pacientes não aidéticos com neurocriptococose que receberam terapia associada AMB e 5FC;}

Grupo II: 48 pacientes aidéticos com neurocriptococose que utilizaram terapia associada $\mathrm{AMB}$ e $5 \mathrm{FC}$;

Grupo III: 38 pacientes aidéticos com neurocriptococose que foram tratados apenas pela AMB.

Os fatores predisponentes, as doenças oportunistas e os antecedentes epidemiológicos e as principais manifestações clínicas foram realçadas, de acordo com uma ficha padronizada, na presente investigação.

\section{Metodologia}

O diagnóstico laboratorial da neurocriptococose foi baseado, principalmente, no método de coloração pela tinta da China e, efetuando-se, a seguir, a contagem do número de células leveduriformes por $\mathrm{mm}^{3}$ na Câmara de Newbauer ${ }^{4} 161820$.

A cultura para Crytococcus neoformans foi realizada em Agar Sabouraud e em Brain Heart Infusion (BHI) em todos os líquidos cefalorraquidianos 116182224 .

Os fatores prognósticos (celularidade, linfócitos e eosinófilos); a glicorraquia; a proteinorraquia; o método da tinta da China e a cultura foram avaliados na evolução da associação $A M B$ e $5 F C$ e da monoterapia $\mathrm{AMB}$, utilizados nos doentes estudados ${ }^{4}$ 782021 .

As possíveis reações adversas, observadas durante os esquemas terapêuticos propostos, foram assinaladas e comparadas nos três grupos selecionados.

\section{Critério clínico}

As doses de $700 \mathrm{mg}$ de $\mathrm{AMB}$ e a de $250 \mathrm{~g}$ de $5 \mathrm{FC}$ foram designadas terapêuticas, respectivamente, para os fármacos empregados nesses pacientes. A partir daí, considerou-se:

remissão: quando os resultados, laboratorial e liquórico, evolutivos retomavam aos valores normais e ausência de manifestação clínica dos doentes avaliados;

recaída ou manutenção: quando os critérios laboratoriais e clínicos não eram satisfeitos isoladamente ou associados.

A morte assinalada antes dessa dose estipulada foi denominada óbito precoce, a partir da dose terapêutica foi designado óbito tardio. Este ocorreu, em média, 1 a 3 anos após a dose terapêutica utilizada.

\section{Análise estatística}

O estudo das associações das taxas nas diversas tabelas foi padronizado pelos testes de Goodman ${ }^{11}$. Para as comparações entre proporções colocaramse letras minúsculas ao lado das respectivas taxas e para as proporções dentro das multinomiais, letras maiúsculas, abaixo, com o objetivo de indicar as diferenças sifnificativas existentes. Todos os testes estatísticos foram efetuados ao nível de $5 \%$ de significância.

\section{RESULTADOS}

\section{Pacientes - dados gerais}

Dos 112 pacientes com neurocriptococose, 86 eram aidéticos. A maioria deles era proveniente da região Centro-Oeste do Brasil e referiam saneamento básico (água e esgoto) em suas residências. Os fatores predisponentes, tais como, etilismo, quimioterapia, diabetes e homossexual, bissexual, droga abusivo, heterossexual, doença transfusional foram, respectivamente, assinalados, em ordem decrescente, nos doentes com neurocriptococose antes e na fase aidética.

Contato com animais (cães e ratos) e aves (pombos, pássaros e galinhas) foram referidos pelos doentes.

As doenças associadas mais encontradas e as principais manifestações clínicas acham-se na Tabela 1.

A evolução aguda ( $<3$ meses) foi observada em 108 dos 112 doentes avaliados. 
Campos EP, Carvalho VO, Marinho SF, Kushnaroff TM, Galvão PAA, Padovani CR. Estudo retrospectivo terapêutico da neurocriptococose em 112 aidéticos ou não. Revista da Sociedade Brasileira de Medicina Tropical 25:241-246, out-dez, 1992.

Tabela 1 - Doenças associadas em 86 doentes dos grupos II e III. Manifestações clínicas em 112 aidéticos ou não, com criptococose.

Doenças

Número de

indivíduos

Pneumocistose

Toxoplasmose

Criptosporidíase

Estrongiloidíase

Tuberculose

Pneumopatias agudas

Sepsis

Herpes simplex

Herpes zoster

Sarcoma de Kaposi

Manifestações clínicas

Respiratórias

Tosse

Dispnéia

Sistema Nervoso Central

Hipertensão intracraniana

Meningoencefalite

Hidrocefalia

Comprometimento

pares cranianos

Irritabilidade

Distúrbio psíquico

Focal

27

17

4

2

22

19

12

18

5

8

$\begin{array}{ccc}\begin{array}{c}\text { Grupo } \\ \text { I }\end{array} & \begin{array}{c}\text { Grupo } \\ \text { II }\end{array} & \begin{array}{c}\text { Grupo } \\ \text { III }\end{array} \\ 1 & 35 & 23 \\ 0 & 5 & 10\end{array}$

\section{Laboratorial}

O diagnóstico etiológico foi realizado pelo método de coloração da tinta da China, seguido pela contagem do número de células encontradas em Câmara de Newbauer, técnicas estas realizadas em todos os líquidos cefalorraquidianos colhidos. A cultura foi positiva para Cryptococcus neoformans nos líquores desses pacientes.

Fatores prognósticos utilizados no estudo liquórico (Tabela2) revelaram:

1. celularidade $\left(<50 \mathrm{cels} / \mathrm{mm}^{3}\right)$ foi comum nos grupos de pacientes aidéticos II e III e rara no grupo I;

2. glicorraquia $(<45 \mathrm{mg} / \mathrm{dl}$ ) foi frequente no inícioe durante, porémnão se revelou diminuída na evolução tardia, análogo ao observado na proteinorraquia ( $>90 \mathrm{mg} / \mathrm{dl}$ ) para os três grupos avaliados;

3. a tinta da China foi significativamente positiva no início e durante a terapia, enquanto o isolamento de $C$. neoformans acompanhou a dose total do fármaco empregado, ou seja, em dose inicial houve mais isolamento que durante o incremento da terapêtutica fúngica;

4. leucopenia, ausência de desvio à esquerda e linfopenia foram assinaladas nos três grupos, sendo de 78 a $83 \%$ respectivamente nos grupos II e III.

Tabela 2 - Fatores prognósticos liquóricos dos três grupos frente à terapia (inicial, 1000mg e $1500 \mathrm{mg}$ ) de anfotericina $B$ associada à 5 fluorcitosina.

\begin{tabular}{|c|c|c|c|c|}
\hline Provas citoquímicas* & $\begin{array}{c}\text { Gru- } \\
\text { po }\end{array}$ & $\begin{array}{l}\text { Ini- } \\
\text { cial }\end{array}$ & $\begin{array}{c}\mathrm{mg} \\
1000\end{array}$ & $\begin{array}{c}\mathrm{mg} \\
1500\end{array}$ \\
\hline \multirow{3}{*}{ Celuraridade $<50$ cels $/ \mathrm{mm}^{3}$} & I & 6 & 15 & 12 \\
\hline & II & 42 & 25 & 15 \\
\hline & III & 33 & 11 & 10 \\
\hline \multirow{3}{*}{ Proteinorraquia $>90 \mathrm{mg} / \mathrm{dl}$} & I & 18 & 7 & 2 \\
\hline & II & 19 & 3 & 2 \\
\hline & III & 14 & 1 & 3 \\
\hline \multirow{3}{*}{ Glicorraquia $<45 \mathrm{mg} / \mathrm{dl}$} & I & 19 & 7 & 8 \\
\hline & II & 31 & 15 & 8 \\
\hline & III & 28 & 12 & 4 \\
\hline \multirow{3}{*}{ Tinta da China + cels $/ \mathrm{mm}^{3}$} & I & 25 & 13 & 9 \\
\hline & II & 45 & 24 & \\
\hline & III & 37 & 11 & O \\
\hline \multirow{3}{*}{ Cultura positiva } & I & 16 & 4 & \\
\hline & II & 34 & 12 & \\
\hline & III & 33 & 7 & 5 \\
\hline
\end{tabular}

* Testes de Goodmam

\section{Terapia comparativa}

1. A $\mathrm{AMB}$ (monoterapia) foi capaz de negativar o esfregaço corado pela tinta da China e o isolamento de $C$. neoformans de modo análogo ao observado na associação AMB e $5 \mathrm{FC}$.

2. Os valores séricos normais, elevados ou 
Campos EP, Carvalho VO, Marinho SF, Kushnaroff TM, Galvão PAA, Padovani CR. Estudo retrospectivo terapêutico da neurocriptococose em 112 aidéticos ou não. Revista da Sociedade Brasileira de Medicina Tropical 25:241-246, out-dez, 1992.

diminuídos do potássio não diferiram significativamente nos diversos momentos da avaliação terapêutica desses grupos.

3. A calemia diminuiu significativamente durante a monoterapia pela AMB. A calemia normal foi significativamente diferente no grupo II nos três momentos ensaiados.

4. A dose terapêutica ( $>700 \mathrm{mg}$ ) de AMB e de (> $250 \mathrm{~g}$ ) de 5FC influenciou de modo significativo à presença dos efeitos colaterais vigentes na associação.

5. A insuficiência renal e a hipocalemia foram significativamente descritas a partir de $700 \mathrm{mg} /$ $\mathrm{AMB}$ e de $250 \mathrm{~g} / 5 \mathrm{FC}$ nos grupos Ie II estudados. Não se verificou tal fato na monoterapia - grupo III.

6. O óbito precoce foi mais evidente no grupo III (monoterapia) enquanto o tardio foi semelhante nos grupos I e III e mais acentuado no grupo II.

7. A remissão e os óbitos ocorridos foram semelhantes nos grupos II e III.

\section{DISCUSSÃO}

\section{Pacientes - aspectos epidemiológicos}

Os achados demográficos, a saber: sexo $(9,3$ M: 1 F); idade média de 35 anos; raça branca predominante; condições sócio-econômicas precátias; fatores predisponentes citados e atividade sexual; homo - $67 \%$ e bissexual - $65 \%$, foram condizentes com os observados na literatura 12345 678910121314151618202324 .

Perez e col $^{18}$ observaram in loco as precárias condições sócio-econômicas das diversas etnias e um aumento real dos toxicômanos nos aidéticos do município de São Paulo. A grande incidência de homossexuais, em nossa casuística, reforçou os estudos realizados por Chuck e col $^{6}$; Mckenzie ${ }^{15}$; Perez e col ${ }^{18}$.

Swinne e col$^{23}$; Perez e col ${ }^{18}$ demonstraram a presença de $C$. neoformans em dejetos de pássaros (87\% a saber: pombos $28,6 \%$; galinhas $28,6 \%$, pássaros $28,6 \%$ e ruas $14,2 \%$ ) e animais residentes na área domiciliar ${ }^{1} 312151617182324$.

\section{Aspectos clínicos}

Os 112 doentes internados, de 3 a 5 meses com neurocriptococose aguda, apresentavam sinais de hipertensão intracraniana, com síndrome meningítica em 95 a $100 \%$ e crises convulsivas focais, respectivamente em 10,20 e $20 \%$ dos avaliados.

Nossos resultados reforçaram os achados referidos por Atkinson e $\mathrm{col}^{2}$; Chuck e $\mathrm{col}^{6}$; Dismukes e $\mathrm{col}^{9}$; Campos e $\mathrm{col}^{5}$; Perez e $\mathrm{col}^{18}$; Sugar e $\mathrm{col}^{21}$. As principais doenças associadas à neurocriptococose em aidéticos foram: pneumocistose (31\%); tuberculose (26\%) e pneumopatias agudas (23\%).

As alterações respiratórias frequentes nos grupos aidéticos II e III e eventuais no grupo I, revelaram a importância e a maior agressão respiratória dessas pneumopatias na neurocriptococose dos aidéticos ${ }^{5}$ 15 .

A alta frequência de Herpes simplex e do sarcoma de Kaposi coincidiu com a presença em nossa casuística, de homo e bissexuais 4561518 .

O óbito precoce nos aidéticos foi mais evidente no grupo III tratados pela monoterapia. Este fato realçou a necessidade da associação $\mathrm{AMB} / 5 \mathrm{FC}$ nas primeiras semanas da internação dos aidéticos com neurocriptococose. A ocorrência mínima de óbito precoce nos grupos não aidéticos permitiu sugerir que a monoterapia e/ou associação propiciaram resultados promissores no tratamento da neurocriptococose desses pacientes 567914182021 .

O óbito tardio foi maior no grupo II - AMB/ $5 \mathrm{FC}$, porém, os resultados foram semelhantes nos grupos I e III. Portanto, a eficácia da monoterapia (AMB) foi semelhante à associação nos não aidéticos, dados esses que divergiram dos de Diamond e $\mathrm{col}^{8}$, porém, similares aos de Chuck e col ${ }^{6}$; Dismukes e $\mathrm{col}^{9}$; Perez e coll ${ }^{18}$.

As grandes modificações dos fatores prognósticos foram observadas nos aidéticos, v.g. Tabela 2, na qual a proteinorraquia sugeriu maior comprometimento cerebral ${ }^{456789}$.

A tinta da China e a cultura positivas (94\%), mostraram tendência à negativação e à persistência da positividade de Cryptococcus neoformans nas doses superiores a $1000 \mathrm{mg} / \mathrm{AMB}$. Campos e $\mathrm{col}^{4}$ relataram elevada positividade e a mínima evidência de gemulação do $C$. neoformans na neurocriptococose de 29 aidéticos. Perez e $\mathrm{col}^{18}$ 
Campos EP, Carvalho VO, Marinho SF, Kushnaroff TM, Galvão PAA, Padovani CR. Estudo retrospectivo terapêutico da neurocriptococose em 112 aidéticos ou não. Revista da Sociedade Brasileira de Medicina Tropical 25:241-246, out-dez, 1992.

observaram relação direta entre o número de leveduras $/ \mathrm{mm}^{3}$ e óbito ocorrido em aidéticos com neurocriptococose. Esses relatos, portanto, coincidiram com os observados na literatura 471218 2224 .

\section{Terapia comparativa}

A monoterapia mostrou-se tão eficaz quanto à associação, de acordo com os resultados observados nos fatores prognósticos (Tabela 2) e na avaliação da terapia comparativa, durante os três momentos considerados na neurocriptococose dos aidéticos estudados 56789131821 .

A monoterapia induziu menor reação adversa $e$ estas foram mais acentuadas a partir de $750 \mathrm{mg} /$ $\mathrm{AMB}$ e de $250 \mathrm{~g} / 5 \mathrm{FC}$ de acordo com as citadas na literatura ${ }^{6781821}$. Além disso, observou-se menor reação adversa nas doses de 0,3 a $0,5 \mathrm{mg} / \mathrm{kg} / \mathrm{AMB}$ e de $130 \mathrm{mg} / \mathrm{kg} / 5 \mathrm{FC}$ associadas na terapia da neurocriptococose $\mathrm{e}^{679}$.

Portanto, a monoterapia inicial (300 a 500mg/ AMB) favoreceu o óbito precoce e, deveria ser evitada, todavia, a partir de $750 \mathrm{mg} / \mathrm{AMB}$ mostrouse a terapia antifúngica ideal. Observou-se que doses acima de $1,5 \mathrm{~g}$ a AMB impediram a recaída. Chuck e $\mathrm{col}^{6}$, no entanto, propuseram a manutenção terapêutica (AMB) como idéia especulativa.

O fluconazol possui boa difusão liquórica e, ao ser comparado com AMB, revelou semelhança na incidência de recaída precoce nos doentes avaliados ${ }^{20}$ 21 , enquanto os demais imidazóis (ketoconazol e itraconazol) não se difundem bem no SNC. Lopez e col ${ }^{14}$ e Pitrak e col ${ }^{19}$ observaram, respectivamente, a instalação da neurocriptococose e da neuroparacoccidioidomicose na evolução terapêutica pelo itraconazol e pelo ketoconazol da micose pulmonar.

A presente investigação revelou que a associação AMB e 5FC inicial, a monoterapia remissiva e de manutenção pela $\mathrm{AMB}$, determinaram menor efeito adverso, menor recaída e óbito tardio nos doentes avaliados.

\section{SUMMARY}

A total of 112 AIDS and no AIDS cryptococcosis patients admitted at Emilio Ribas Hospital - São Paulo, Brazil, were treated with amphotericin $B(A M B)$ or amphotericin $B$ and 5 fluorcytosine (5FC). Age, race, predisposing and epidemiological factors, respiratory symptons were evaluated. Goodman tests applied in three patients groups $(I, I I$ and III) with associate or unique therapy revealed: 1. prognosticfactors: leukocytes ang glucose showed similar response in groups I, II and III and protein spinal fluid after $1.5 \mathrm{~g} / \mathrm{AMB} ; 2$. India ink tests and Cryptococcus culture were often positive until 1. Og/AMB; 3. significant hypokalemia during monotherapy. Hypo and hyperkalemia had similar data in associate therapy; 4. significant difference in adverse reactions often appeared above $0.7 \mathrm{~g} \mathrm{AMB} / 250 \mathrm{~g} 5 \mathrm{FC} ; 5$. early and late death were common in group III (unique) and group I (no AIDS) and III (2.5 to $4.0 \mathrm{~g})$ respectively; 6. similar remission and deaths were verified in AIDS/ cryptococosis. Conclusions: adverse reactions were observed above $0.75 \mathrm{~g} / \mathrm{AMB}$ plus $250 \mathrm{~g} 5 \mathrm{FC}$. Association was important in initial therapy and $A M B$ maintenance permitted late relapses.

Key-words: Neurocryptococcosis. AIDS and no AIDS. Therapy.

\section{REFERENCES}

1. Ajello L. Occurence of Cryptococcus neoformans in soils. American Journal of Hygiene 67:72-77, 1958.

2. Atkinson W, Troxler S, McFarland L. Extrapulmonary crytococcal disease as manifestations of AIDS in Lousiana. In: Abstracts of International conference of AIDS, Montreal p.484, 1989.

3. Bennett JE, Kwon-Chung KJ, Howard DH. Epidemiological differences between the two varieties of Cryptococcus neoformans. American Journal of Epidemiology 120:123-130, 1977.

4. Campos CEOP, Santos NNQ, Takahashi MM, Astone E, Ayrosa Galvão PA, Campos EP. Cryptococcus neoformans meningitis (CNM) in Hospital Emílio Ribas - São Paulo, Brazil. In: Abstracts of XI Congress of the International Society for Human and Animal Mycology, Montreal p.82, 1991.

5. Campos EP, MarinhoSF, Carvalho VO, Kushnaroff TM, Ayrosa Galvão PA. Clinical therapy sutdy: 110 AIDS and no AIDS cryptococcosis. In: Abstracts of International Conference on Cryptococcus and Cryptococcosis. Jeruzalem p.5, 1989.

6. ChuckSL, Sande MA. Infections with Cryptococcus neoformans in acquired immunodeficiency syndrome. New England Journal Medicine 321:794799, 1989.

7. Diamond RD. Cryptococcus neoformans. In: Mandell GL, Douglas Jr RG, Bennett JE (eds). Clinical and 
Campos EP, Carvalho VO, Marinho SF, Kushnaroff TM, Galvão PAA, Padovani CR. Estudo retrospectivo terapêutico da neurocriptococose em 112 aidéticos ou não. Revista da Sociedade Brasileira de Medicina Tropical 25:241-246, out-dez, 1992.

practice of infectious diseases, $2^{\text {nd }}$ edition, John Wiley, New York p.1981, 1990.

8. Diamond RD, Bennett JJE. Prognostic factors in cryptococcal meningitis. A study in 111 cases. Annals of Internal Medicine 80:176-181, 1974.

9. Dismukes WE, Cloud G, Galtes HA, Kerkering TM, Medoff G, Blackweder WC. Treatment of cryptococcal meningitis with combination Amphotericin B and Flucytosine for four as compared with six weeks. New England Journal of Medicine 317:334-398, 1987.

10. Gonçalves AJR, Rosembaun R, Wanke B, Vieira W. A criptococose no Estado do Rio de Janeiro. Apresentação de 10 casos e revisão da literatura fluminense. Arquivo Brasileiro de Medicina 65:395398, 1988.

11. Goodman LA. Simultaneous confidence intervals for contrast among multinomial populations. Annals of Matematical-Statistic 35:716-775, 1964.

12. Howard DH, Chung KJ. The ecology and epidemiology of neoformans and gattii of Cryptococcus neoformans. In: Abstracts of International Conference on Cryptococcus and Cryptococcosis. Jeruzalem p.S-2, 1989.

13. Kovacs JA, Kovaks AA, Polis M, Wright C, Gill VJ, Bennett JE. Cryptococcosis in the immunodeficiency syndrome. Annals of Internal Medicine 103:533$583,1985$.

14. Lopez DLR, Marinho SF, Cirino EMI, Ayrosa Galvão PA, Campos EP. Itraconazol em Aidético com Criptococose. In: Resumos do XXVICongresso da Sociedade Brasileira de Medicina Tropical, Natal p.79, 1990.

15. Mckenzie R, Travis WD, Dolan SA, Pittaluga $S$, Yarchoan R, Masur $H$. The causes of death in patients with human immunodeficiency virus infection. A clinical and pathologic study with emphasis on role of pulmonary diseases. Medicine
70:326-343, 1991.

16. Morales Calvo BM. Antígeno capsular, variedades y serotipos de Cryptococcus neoformans en pacientes con imunodeficiencia adquirida y neurocriptococcosis en São Paulo - Brasil. Tese de mestrado, Escola Paulista de Medicina, São Paulo, SP, 1989.

17. Negroni P. Cryptococcosis. In: Micosis profundas (cutaneas y visceralis). Buenos Aires. Comission de Investigacion Cientifica III:13-47, 1966.

18. Perez LPR, Bertrand A, Melhem MSC, Giudice MC, Pires MFC, Grosco SFR, Campos EP, Ayrosa Galvão PA, Lahamann M. Cryptococcosis in HIV-1 infections: clinical, laboratory and epidemiological study. In: Abstracts of XI Congress of International Society for Human and Animal Mycology, Montreal p.99, 1991.

19. Pitrak DL, Burton R, Andersen BR. Cerebral blastomycosis after ketoconazole therapy for respiratory tract blastomycosis. Annals Journal of Medicine 86:713-714, 1989.

20. Rippon JW. Cryptococcosis. In: Medical Mycology: The pathogenic Actinomicetes. $3^{\text {rd }}$ edition Sanders. Philadelphia p.582-609, 1988.

21. Sugar AM, Stern JJ, Dupont B. Treatment of cryptococcal meningitis. Review of Infectious Diseases 12 (supl.3):338-348, 1990.

22. Staib F. Cultural diagnosis of Cryptococcus neoformans and cryptococcosis. In: Abstracts of International Conference on Cryptococcus and Cryptococcosis. Jeruzalem p.S-16, 1989.

23. Swine D, Nkurikiyinfura JR, MuyembeTL. Clinical isolate of Cryptococcus neoformans from Zaire. Journal of Clinical Microbiology 5:50-51, 1986.

24. Takahashi M, Corez RN, Pereira AD, Huggins DW, Lacaz CS. Isolamento de Cryptococcus neoformans das fezes do pombo, do solo e ninho de pombos. Revista Brasileira de Medicina 44:6-9, 1987. 